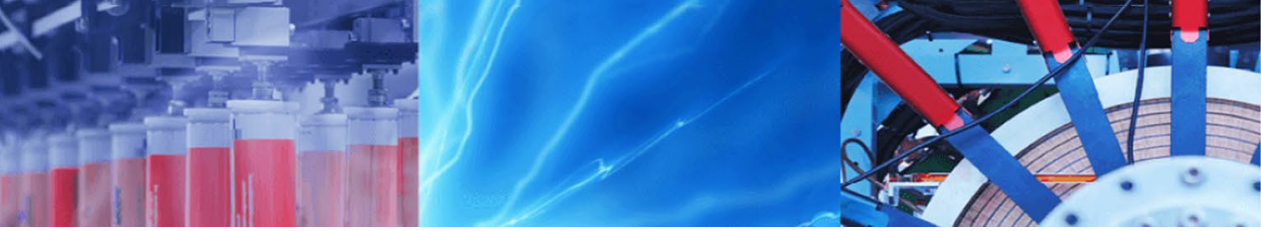

Research Article

\title{
Alarm detection and monitoring in industrial environment using hybrid wireless sensor network
}

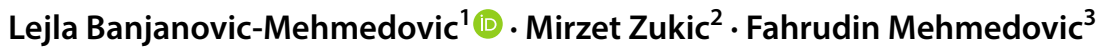

(c) Springer Nature Switzerland AG 2019

\begin{abstract}
Cyber-physical production systems, relying on the latest development of computer science, information and communication technologies, manufacturing science and technology are leading the way to a new industrial age, defined by the fourth industrial revolution. In this paper we present alarm detection in the industrial environment using a hybrid wireless sensor network (HWSN). As wireless static sensors are highly limited in terms of sensing, computation, communication, battery life, and the actions they can perform, our framework includes an autonomous mobile robot as an integral part of HWSN. We propose the mobile robot navigation technique based on sensor nodes as tags attached in the robot's environment to define a target point for a mobile robot inspection task. The experimental scenarios presented in this paper are provided to illustrate the effectiveness of alarm detection using the wireless sensor network with mobile robot inspection.
\end{abstract}

Keywords Alarm detection · Cyber-physical systems · Industry 4.0 Mobile robot inspection · Smart factory $\cdot$ Wireless sensor networks

\section{Introduction}

Cyber-physical systems (CPS) are enabling technologies which bring computational and physical worlds together to create a networked world in which intelligent objects communicate and interact with each other. Cyber-physical systems represent the next evolutionary step from existing embedded systems, which together with the internet and data and available services form cyber-physical systems [1].

Cyber-physical production systems, relying on the latest development of computer science, information and communication technologies, manufacturing science and technology are leading way to a new industrial age, defined by fourth industrial revolution [2]. Industry 4.0 represents a leap forward from more traditional automation to fully connected and flexible systems, based on the application of artificial intelligence (Al) and increasing sophistication of cyber-physical systems [3].

The Industry 4.0 consists of several technological areas, which are horizontal and vertical system integration, the internet of things, cyber security, the cloud, big data analytics, simulation, additive manufacturing (3D printing), augmented reality and robot [4]. The concept Industry 4.0 is best defined by the project's "smart factory" through the merging of virtual and physical worlds through cyber-physical systems and the resulting fusion of technical and business processes [5]. The smart factories require the underlying processes and materials to be connected through a transparent network with real-time tracking and monitoring in order to generate the data necessary to make real-time decisions. An optimized

Lejla Banjanovic-Mehmedovic, lejla.banjanovic-mehmedovic@fet.ba; Mirzet Zukic, mzukic@capeannenterprises.com; Fahrudin Mehmedovic, fahrudin.mehmedovic@hr.abb.com | ${ }^{1}$ Faculty of Electrical Engineering, University of Tuzla, 75000 Tuzla, Bosnia and Herzegovina. ${ }^{2}$ Cape Ann Enterprises, 75000 Tuzla, Bosnia and Herzegovina. ${ }^{3} \mathrm{ABB}$ Representation for Bosnia and Herzegovina, 75000 Tuzla, Bosnia and Herzegovina.

SN Applied Sciences (2019) 1:263 | https://doi.org/10.1007/s42452-019-0269-y 
smart factory allows operations to be executed with minimal manual intervention and high reliability. In order to minimize failures of manufacturing processes and avoid huge economic losses, it is important to act before issues or challenges arise, rather than simply reacting to them after they occur. This feature can include regularly inspected activity, identifying anomalies and monitoring safety concerns [5].

As abnormal events can happen at uncertain time, more sensor nodes are needed in the industrial environment to set an alarm precisely. Harsh environment conditions in the petroleum or chemical industry as well as in nuclear plants is essential for introducing sensor networks. The sensor networks have recently emerged as an effective tool for monitoring and detecting alarms states in industrial environments, especially wireless sensor networks [6, 7]. In order to deploy the sensor networks in such large environments, often with hundreds of nodes, the wireless sensors must be low-cost and affordable. Hence, the wireless sensors are typically highly limited in terms of sensing, computation, communication, battery life and the actions they can perform. These limitations can be addressed through addition of more capable mobile robot sensors to form a hybrid wireless network. Higher-capability mobile robots may be dispatched to gather more accurate temperature or humidity readings, or recharging a static sensor battery which the static sensors are not equipped for. Although the static sensors are less capable than mobile robots, they are also much less expensive and can be used to cover a vast area at a low cost. The static sensors must assign these events to mobile robots while simultaneously minimizing the distance travelled by robots and communication among the static sensors to prolong their battery life [8].

There are few examples of hybrid sensors networks in different task allocation. The event assignment problem for assigning mobile robots to events in hybrid wireless sensor networks is presented in [8]. The achievements in the field of electric power substation equipment inspection by mobile robot are presented in [9]. Intruder detection by using both the WSN and mobile robots is presented in [10]. The paper [11] addresses the problem of tracking mobile robots using Kalman filter in indoor wireless sensor networks (WSNs). The wireless sensor nodes collect RSSI information sent by mobile robots. A range-based data fusion scheme is used to estimate the robot's trajectory. The complete integration of the sensor-equipped robots into the WSN system to visualize sensed data information into 3D graphical control interface for advanced planning and execution against any anomalies detected in different environment is presented in [12]. An efficient system that employs a smaller number of stationary nodes that collaborate with a small set of mobile nodes in order to improve the sensor area coverage and event detection is presented in [13].

In this paper, we focus on the alarm detection and monitoring of smart factory environment using the hybrid wireless sensor network. As abnormal events can happen at uncertain time, more sensor nodes are needed in the industrial environment to set an alarm precisely. Our framework includes the autonomous mobile robot as an integral part of the wireless sensor network, assigned for an inspection task.

We first present an overview of alarm detection techniques in Sect. 2. The architecture of cyber-physical system is presented in Sect. 3. Section 4 presents a brief review of mobile robot navigation techniques and our mobile robot inspection approach using sensor nodes as tags. The results of this research are presented in Sect. 5. Finally, we summarize our findings in Conclusion.

\section{Alarm detection techniques}

In factories, manufacturing processes depend on corrected manufacturing or operational environment values at all stages of the work. To prevent the accidents in a factory, a key method to be taken is to effectively monitor the certain physical values (temperature of the environment or equipment such as transformers, cable connectors, leakage detection, radiation check, intrusion notification, etc.). Additional recent data analytics capabilities include event stream processing and complex event processing in critical operating plant situations [14]. An abnormal situation or anomaly is defined as a disturbance or series of disturbances in a process that cause plant operations to deviate from their normal operating or overrun limits. The term anomaly detection can be used to refer to both the detection of causes based on system changes and component fault. An alarm system comprises hardware and software components, which can signal an alarm state, transmit the signal to the process automation system, record the signal, and display a message about the signal to the operator (monitoring).

There are many event detection techniques [15]. The initial idea of event detection is to use a threshold value. If the value of sensor node exceeds the pre-defined alarm 
threshold, an event is considered to happen. Pre-alarm function is a programmable option which determines a response of the system to a real-time detector sensing values above the programmed setting as an early warning of incipient or potential abnormal conditions. The modelbased event detection technique models event phenomena in a specific form such as mathematical formulas or maps. Pattern matching-based event detection methods define a data pattern for an event, and then an event is detected if the data pattern matches with the event pattern. Fuzzy logic is a kind of machine learning which can tolerate an unreliable and imprecise sensor reading. In cases where the behavior of a system is either unknown or too complex, the use of artificial neural networks (ANN) is more attractive as an alternative data-based solution. In last time, there are many applications of ANN implementations to solve anomaly detection issues [16].

In our research, we used pre-alarm detection and alarm detection techniques in the hybrid wireless sensor network for cyber-physical system monitoring. This situation allows the possible cause of the pre-alarm to be investigated prior to the full alarm condition. The pre-alarm signal also provides an opportunity to filter out false alarms.

\section{Proposed architecture of hybrid wireless sensor network}

Wireless Sensor Network (WSN) includes a number of battery powered sensor nodes, a processing subsystem for local data processing, memory and a wireless communication module [17]. Each individual node in the network can monitor its local region and communicate through a wireless channel with other nodes to collaboratively produce high-level representation of the environment states.

Standard family for industrial wireless sensor networks application is IEEE802.15 standard group. IEEE 802.15.4/ ZigBee is a low-cost wireless sensor network with data-rate $(250 \mathrm{~Kb} / \mathrm{s})$. Zig Bee transmits the data on 10-75 meters, depend on environment and application and can operate in the unlicensed RF worldwide $(2.4 \mathrm{GHz}$ global, $915 \mathrm{MHz}$ Americas or $868 \mathrm{MHz}$ Europe). It connects thousands of sensors in a factory for transfer sampled process data to control systems. The topologies of ZigBee are tree, mesh, or star and it depend on application and operation. WirelessHART (Highway Addressable Remote Transducer Protocol) is an industrial standard of wireless technology. It is a low cost and low speed solution for plants in industries operations. The data-rate of WirelessHART is $250 \mathrm{~kb} / \mathrm{s}$ and use $2.4 \mathrm{GHz}$ ISM radio band with 15 channels and centralized architecture. ISA-100 is a family of standard for industrial automation and control applications based on wireless sensor technologies. The architecture of ISA-100 system includes thousands of devices in the range of $1 \mathrm{~km}$ in a plant. WiFi-IEEE802.11 standard is for local area networks and use in various environments. It is a high speed, moderate range Ip data transfers in local area networks. UWB (Ultra-Wideband) technology is a short-range wireless communication with low power consumption. The data rate must be $100 \mathrm{Mb} / \mathrm{s}$ to satisfy the industrial use [18].

Those wireless static sensors are typically highly limited in terms of sensing, computation, communication, battery life, and the actions they can perform. These limitations can be addressed through the addition of more capable mobile robot sensors to form the hybrid wireless network [8]. In general, static sensors detect events which must be handled by mobile robots. These events are associated with a space point where a mobile robot performs a task, such as gathering a more accurate temperature reading, or recharging a static sensor battery or another specific task. The static sensors must assign these events to the mobile robots while simultaneously minimizing the distance travelled by the robots and the communication among the static sensors to prolong their battery life.

The designed system in our research was principally based on the hybrid wireless sensor network (HWSN). Most of the sensor nodes act as terminal devices, can only collect data and send it to the coordinator node. The mobile robot is a coordinator of this hybrid wireless sensor network. The system framework is shown in Fig. 1.

Each static node contains an Arduino Atmega 328 processing unit, a BMP180 sensor for temperature and pressure measuring and a communication module. The communication between the nodes was established through a wireless communication using the XBee module and the ZigBee/IEEE 802.15.4 standard. XBee radio supports the AT and API mode for sending and receiving data [19]. The Xbee modules used the AT mode for this application.

In our approach, the mobile robot is assigned to static sensors to handle the events surrounding them. In case that the static sensors allocate events (temperature or pressure alarm detection), they send this information to the mobile robot which immediately starts the inspection task toward the target point in the industry environment where the event was detected. 
Fig. 1 Architecture of the designed hybrid wireless sensor network

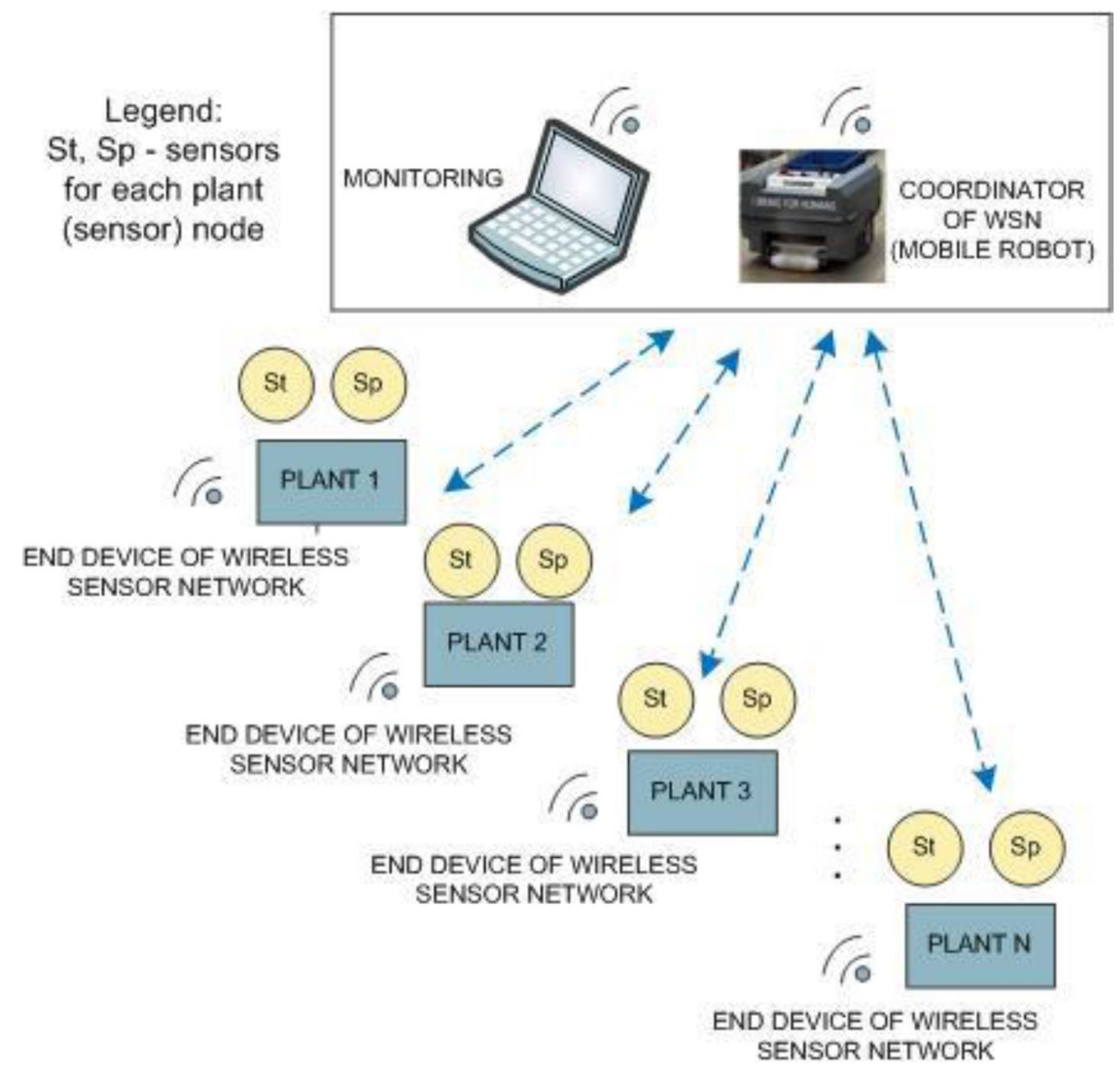

\section{Mobile robot inspection}

The robot navigation methods generally fall under one of the following categories: dead-reckoning-based, landmark-based, vision-based, map-based and behavior-based techniques [20]. The idea of dead-reckoning navigation systems is the integration of incremental motion over time. This navigation method is based on continuous encoder readings that provide the position, orientation, and linear and angular velocities of the robot. This type of navigation is widely used due to its simplicity and ease of maintenance. However, small precision errors and sensor drifts lead to increasing cumulative errors in the robot's position and orientation, unless an independent reference is periodically used to correct the error.

The vision-based navigation improves the robot position estimation by tracing visual features in the environment and using them as landmarks. This measurement usually returns bearing to the visual features only, with no priori knowledge of the landmark positions. Such technique also has its own disadvantages, which include the lack of

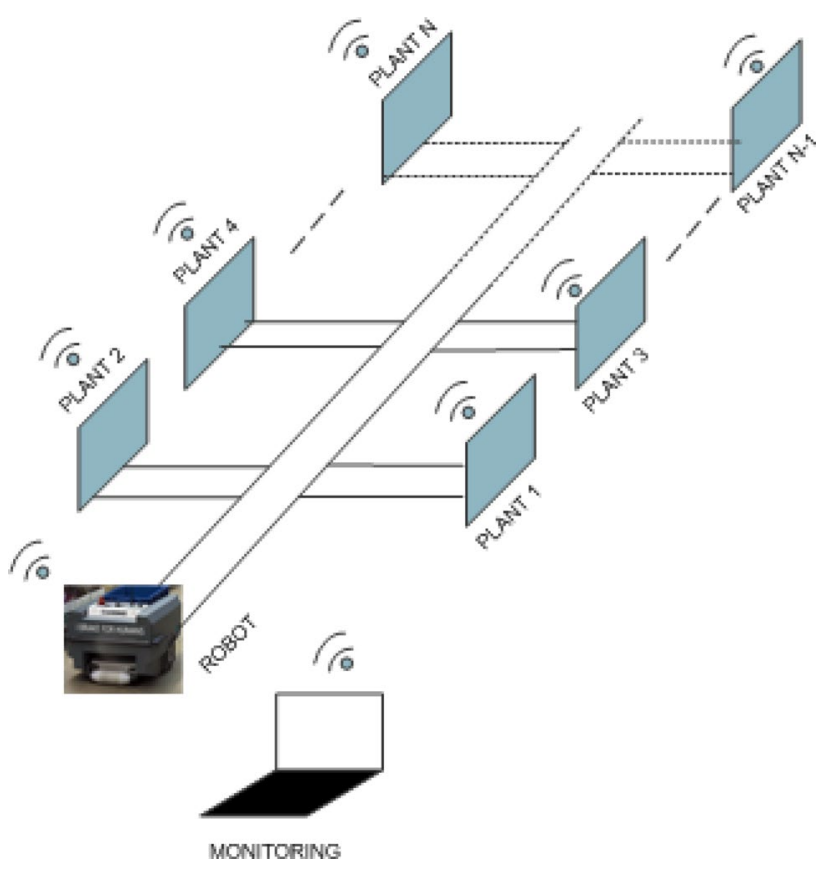

Fig. 2 Sensor-node based navigation in industry pilot platform 


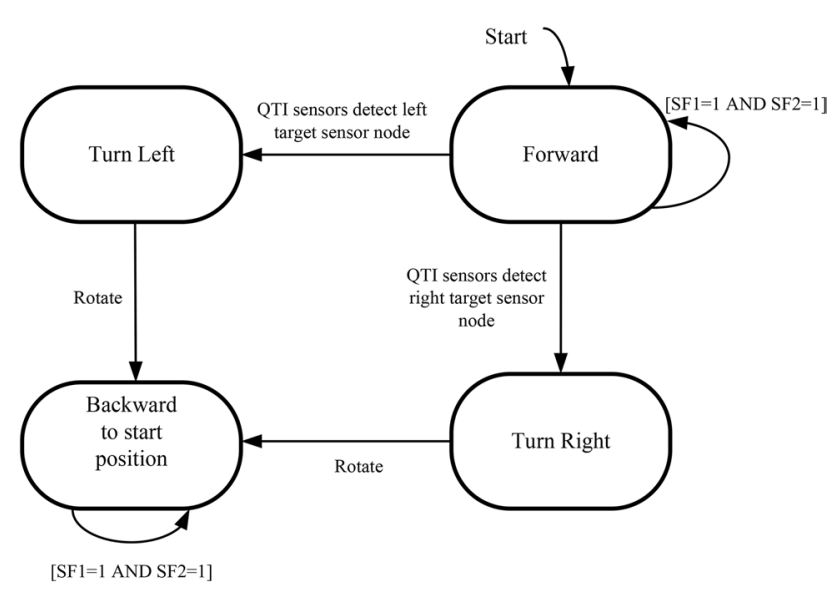

Fig. 3 Finite state machine (FSM) of mobile robot navigation in industry pilot platform

information depth, complex image processing algorithms with high computation and its dependence on the working environment. This problem can be exterminated by adopting the behavior-based navigation systems, as they can incorporate a relatively large number of sensors, making them suitable for navigation in unstructured environments. However, relying on numerous sensors makes the system vulnerable to their drifts and cumulative errors. To overcome this shortcoming, some researchers used artificial landmarks to compensate for these errors. For example, in some studies, RFID tags were placed in particular locations in the workspace, and the robot was equipped with an RFID reader to communicate with the tags to estimate its position.

Many existing methods for robust mobile robot navigation employ vision or sonar or IR sensors, however, these often have problems with reliability [21]. The possible solution is the use of radio frequency identification (RFID), with which is possible to dynamically calculate the robot's position and navigate autonomously with precise localization, even without all of the aforementioned sensors. The paper [22] described an intelligent navigation methodology of a nonholonomic mobile robot using RFID which offers an alternative to various kinds of the navigation strategy, such as the vision system, topological map and sensor fusion etc., which have been currently employed in this field. This study provides a solution that employs the use of received signal strength indication of RFID tags in the $2 \mathrm{D}$ plane based on which the robot plans its path in its $2 \mathrm{D}$ workspace.

Topological maps are typically represented as graphs in which navigation-relevant places are modeled as graph nodes and connections between places indicated by graph edges [23].
Fig. 4 Laboratory experimental setup for alarm detection in plant 4

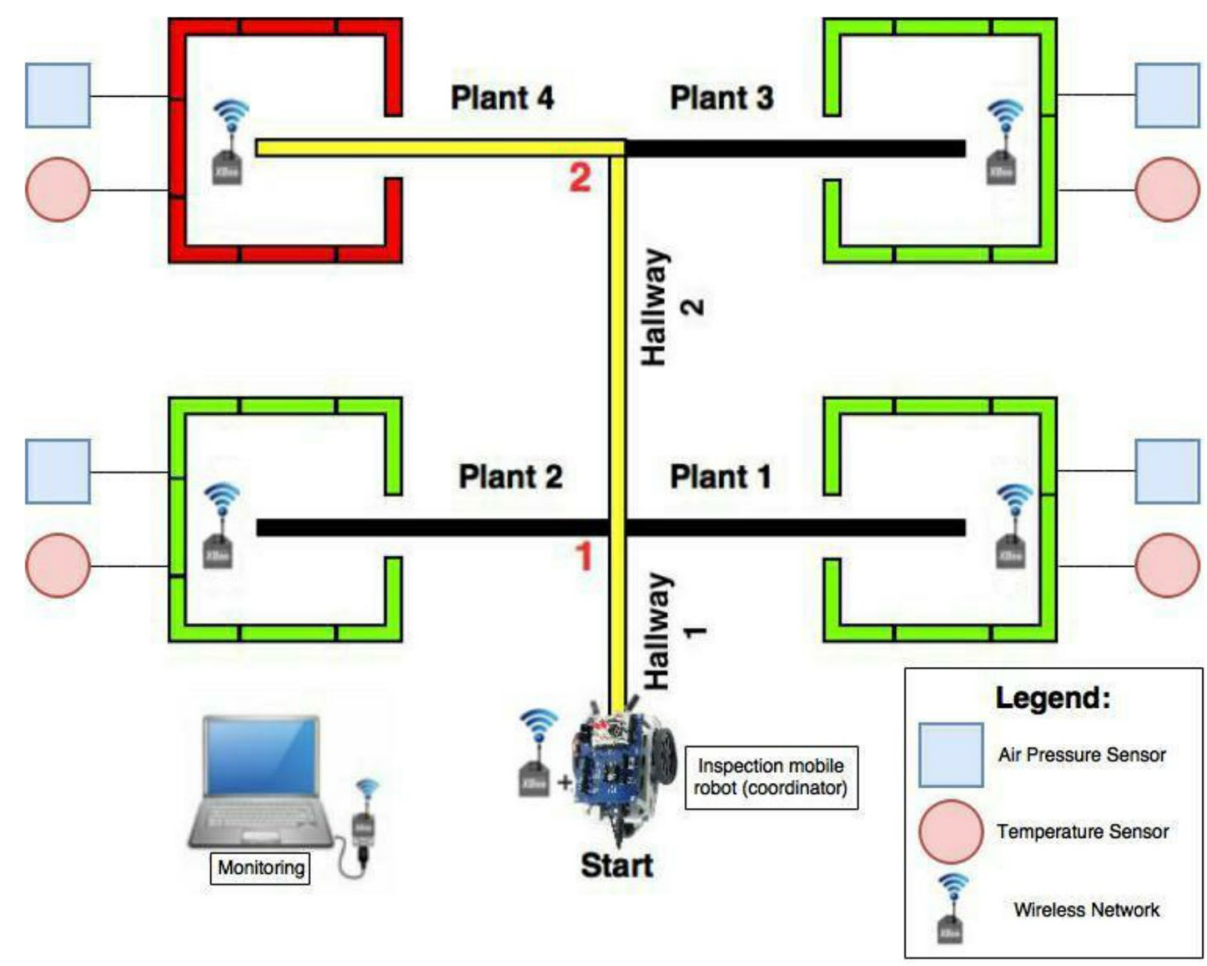

SN Applied Sciences a SPRINGER NATURE journal 


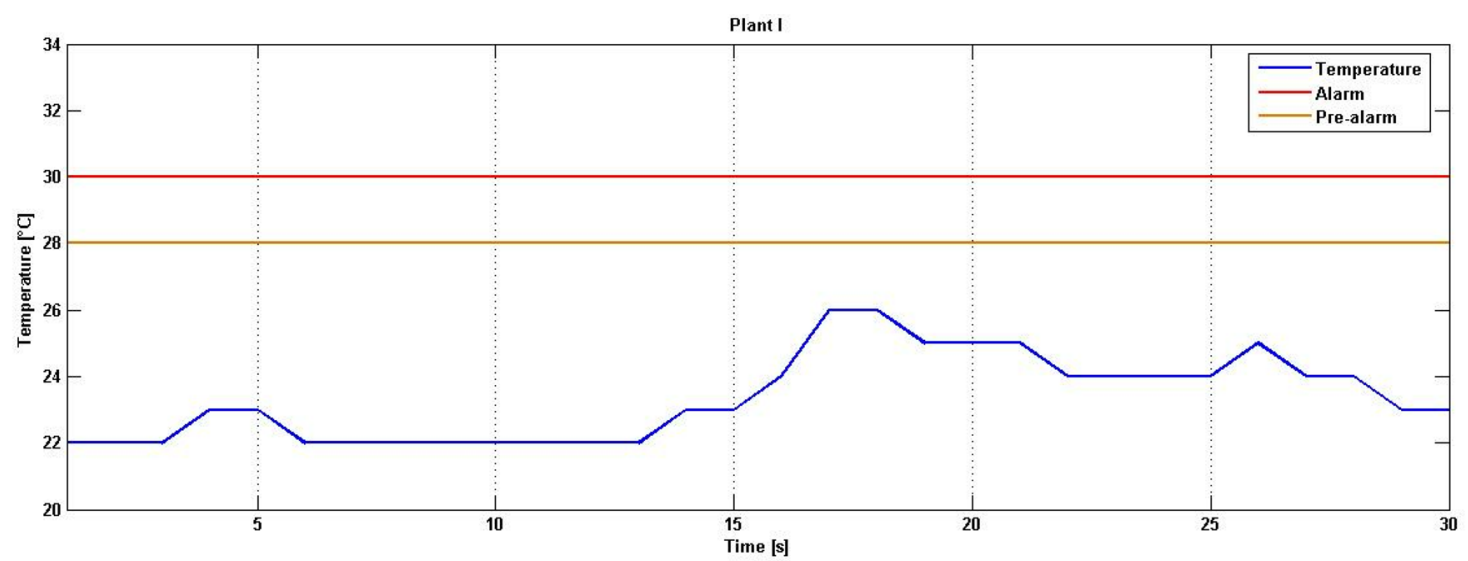

(a)

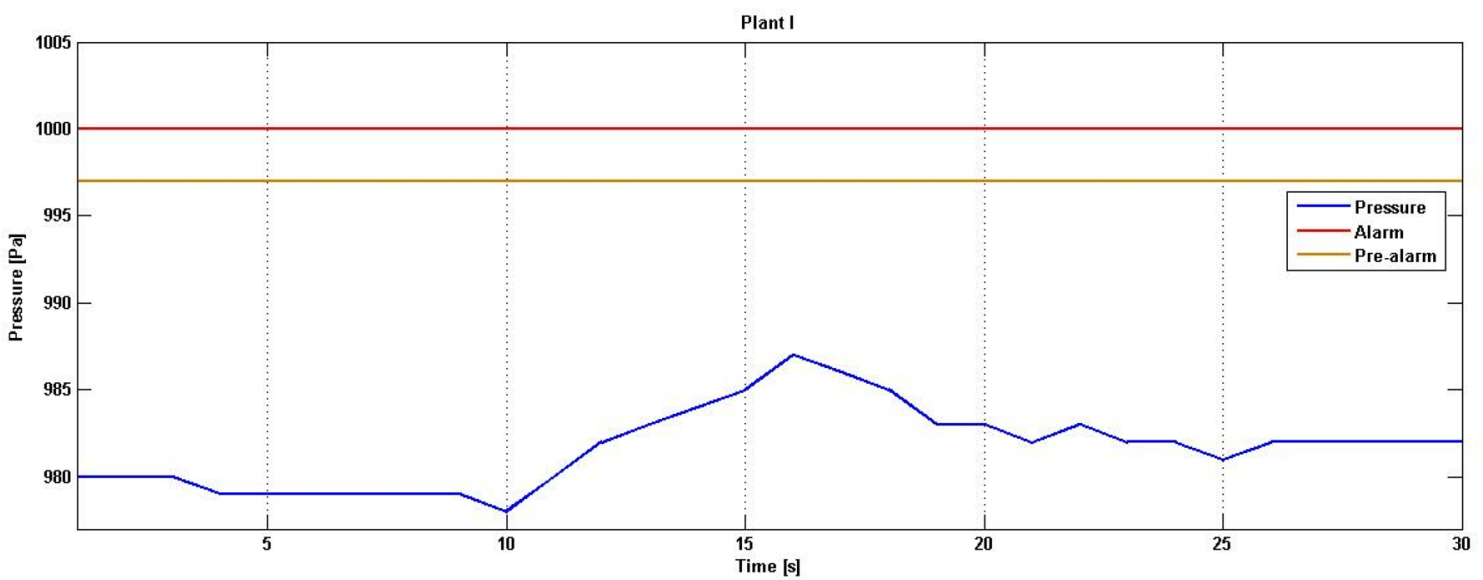

(b)

Fig. 5 Measuring for the selected sensor node (plant 1): $\mathbf{a}$ temperature and $\mathbf{b}$ pressure

In this paper, the wireless sensor nodes are mounted as tags at fixed locations in the 2-D space. The mobile robot followes lines in the industry environment toward the target point (sensor node) where the alarm is detected. Using different combination of QTI infrared emitter/receiver sensors, the mobile robot can detect intersection points in the path and change states. The mobile robot inspection task for our experimental setup is presented in Fig. 2 .

In case, when the inspection mobile robot gets information from the perception unit, it makes possible actions D_ $i=\{$ Forward, Left, Right, Rotation 180, Back\}. It moves forward through the hallway between the intersection points (topological nodes) and right or left toward the plant point (target sensor node). After inspection the certain plant with anomaly detection, the mobile robot rotates and goes back to hallway and toward start position. The finite state machine (FSM) of mobile robot navigation is presented in Fig. 3 .

\section{Alarm detection and monitoring of industry environment pilot platform}

The idea of experimental presentation of alarm detection and monitoring of the industry environment is to organize four sections inside an industrial plant, two on the right and two on the left side, with two corridors among them. Several tests were performed to validate the alarm detection using 


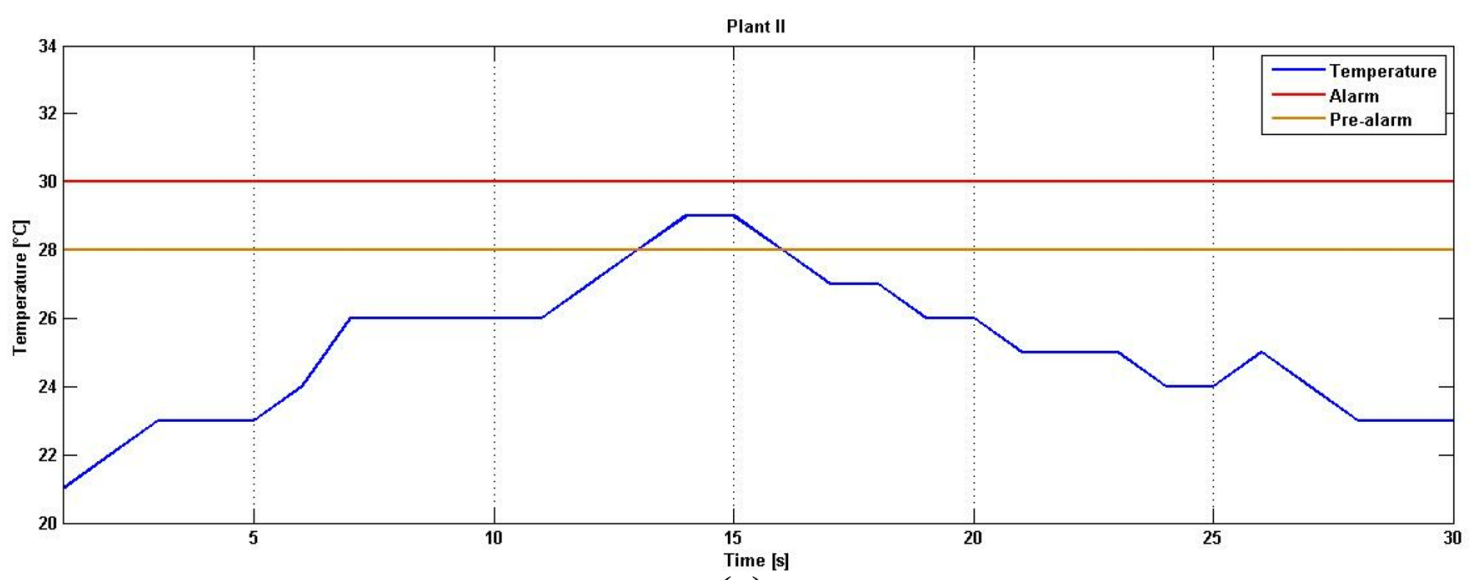

(a)

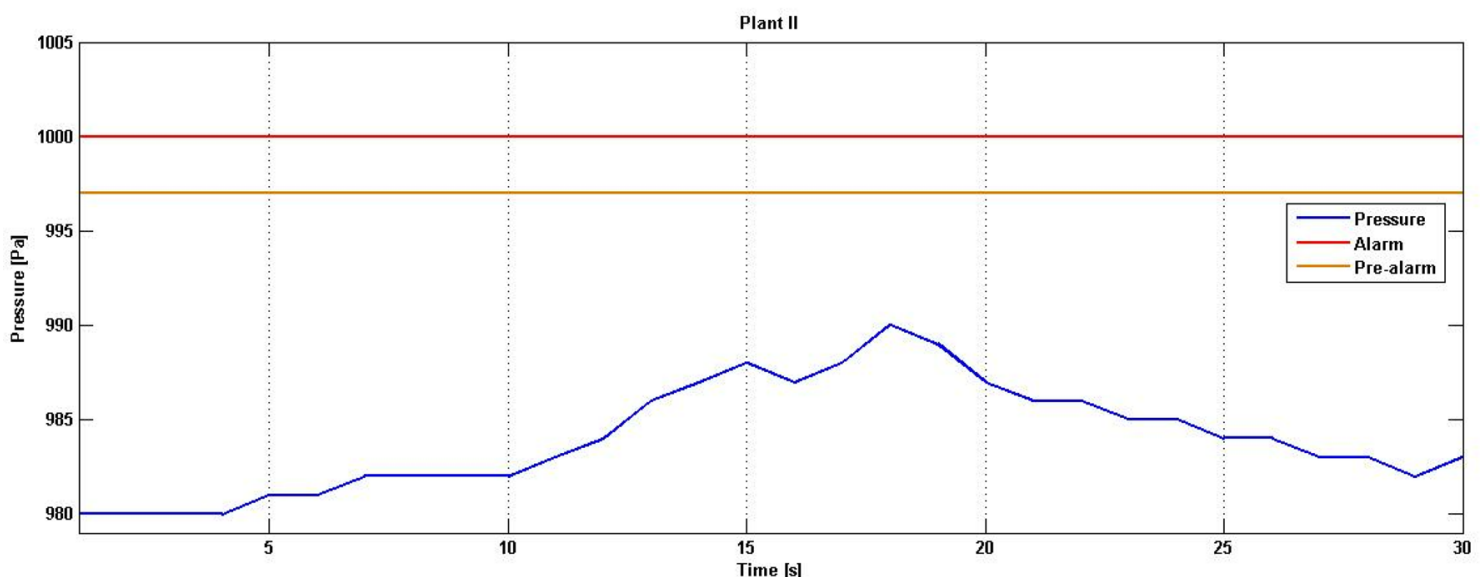

(b)

Fig. 6 Measuring for the selected sensor node (plant 2): a pre-alarm temperature and $\mathbf{b}$ pressure

the hybrid wireless sensor network in the laboratory experimental setup. One example of an experimental scenario is presented in Fig. 4.

Figures 5, 6, 7 and 8 show the results of a measured level of temperature and pressure for the selected wireless sensor nodes. Pre-alarm detection is presented in Fig. 6 and alarm detection for temperature in Fig. 8.

The inspection robot states in the environment during a navigation process are presented in Fig. 9. The navigation target point is defined with the sensor node, where we have information that alarm was detected.
This work proposes an intelligent monitoring system suitable for factory area control using hybrid wireless sensor network. A smart factory health monitoring system was created to improve industrial processes by reducing the handling of dangerous things. The operator can monitor status of static nodes and mobile robot via the intelligent monitoring system instead of onsite manual inspecting. Unlike conventional alarm methods, these systems monitor and control the capabilities of each alarm initiating and signaling device through static sensor node and system software. In effect, 


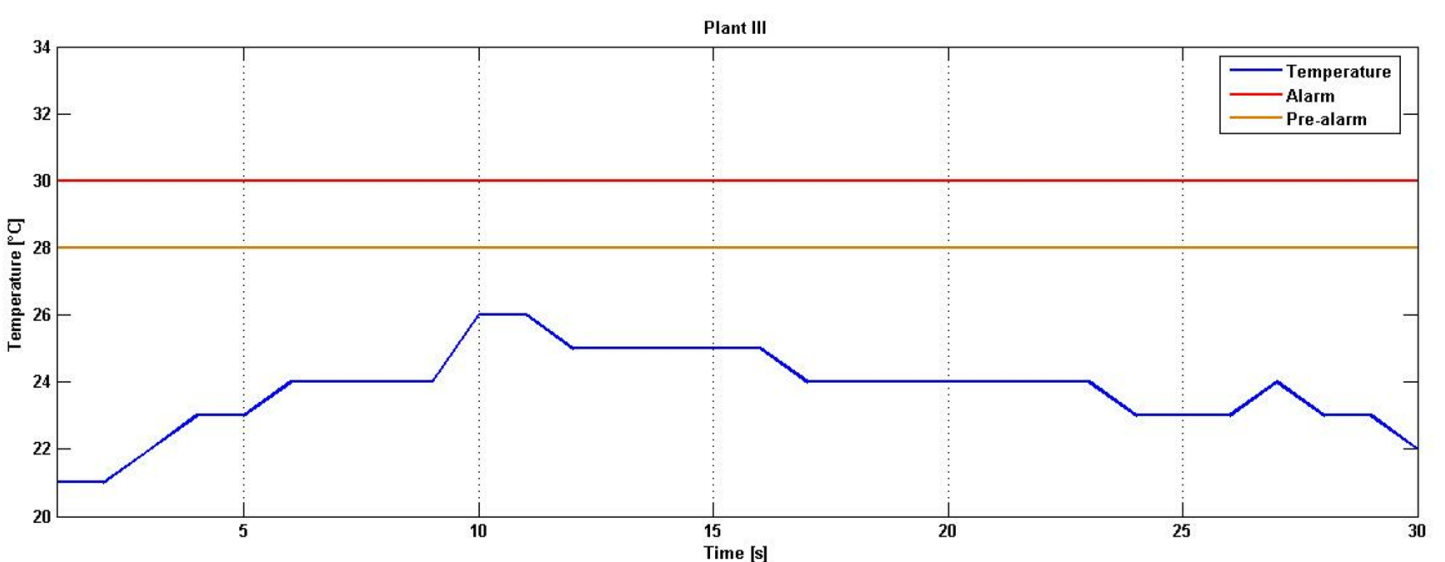

(a)

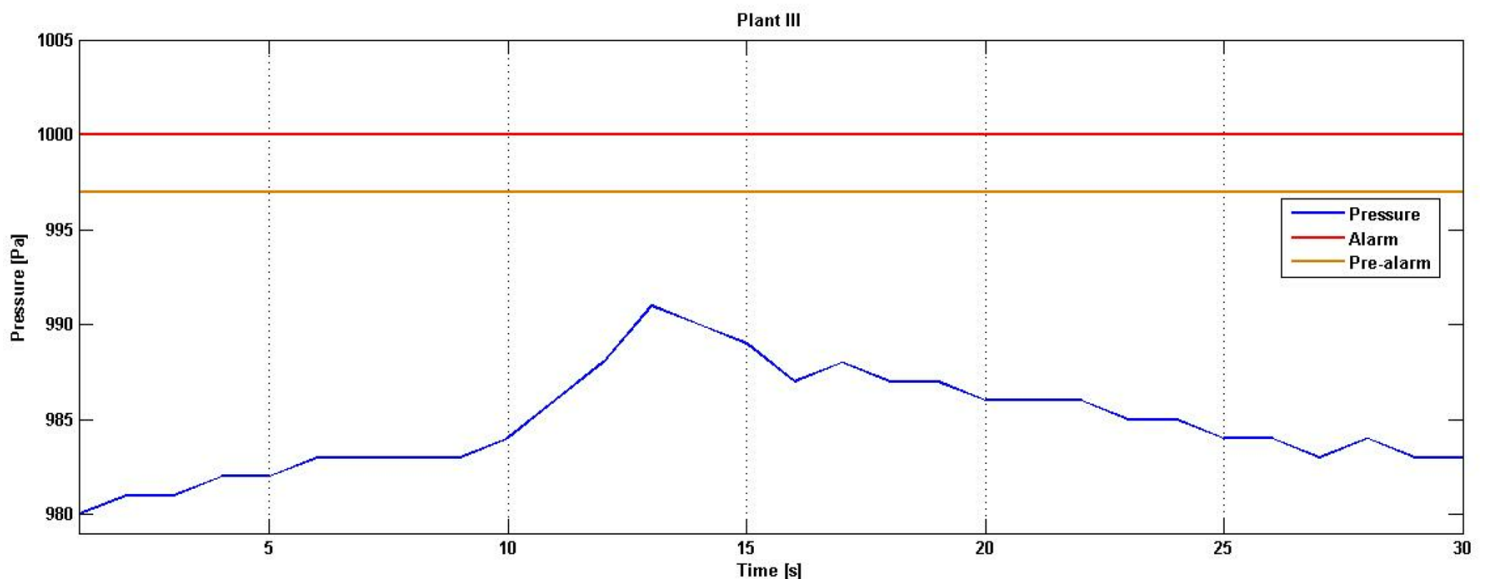

(b)

Fig. 7 Measuring for the selected sensor node (plant 3): $\mathbf{a}$ temperature and $\mathbf{b}$ pressure

each intelligent sensor node is a small computer overseeing and operating a series of input and output devices.

The graphical based monitoring system based on the wireless sensor network for one example of the alarm detection scenario is presented in Fig. 10. Microsoft Visual Studio is used for realization of graphical monitoring, which can display the measuring data collected by wireless sensor nodes in real-time and the inspection robot state. The control panel can give three different visual indications: for stable state, green indication (if the temperature and pressure are lower than the pre-alarm limit); pre-alarm state (orange indication) and alarm state (red indication) for each sensor node. This situation allows the pre-alarm to be investigated prior to a full alarm condition. The pre-alarm signal also provides an opportunity to filter out false alarms. 


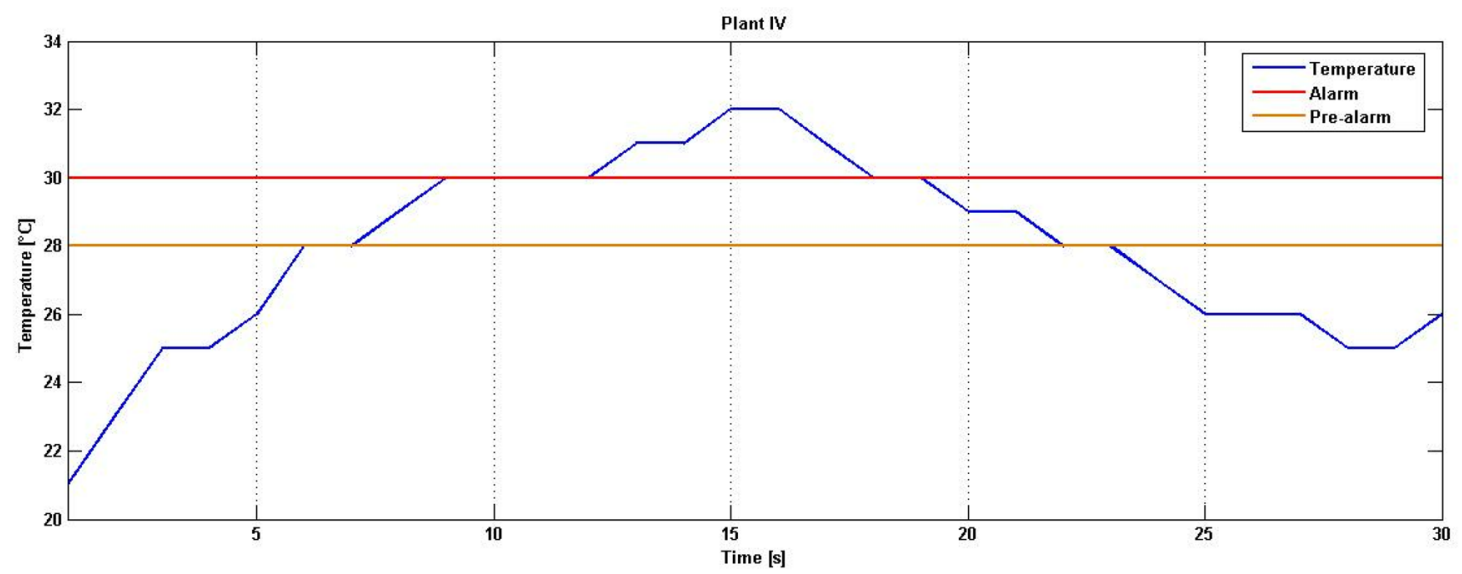

(a)

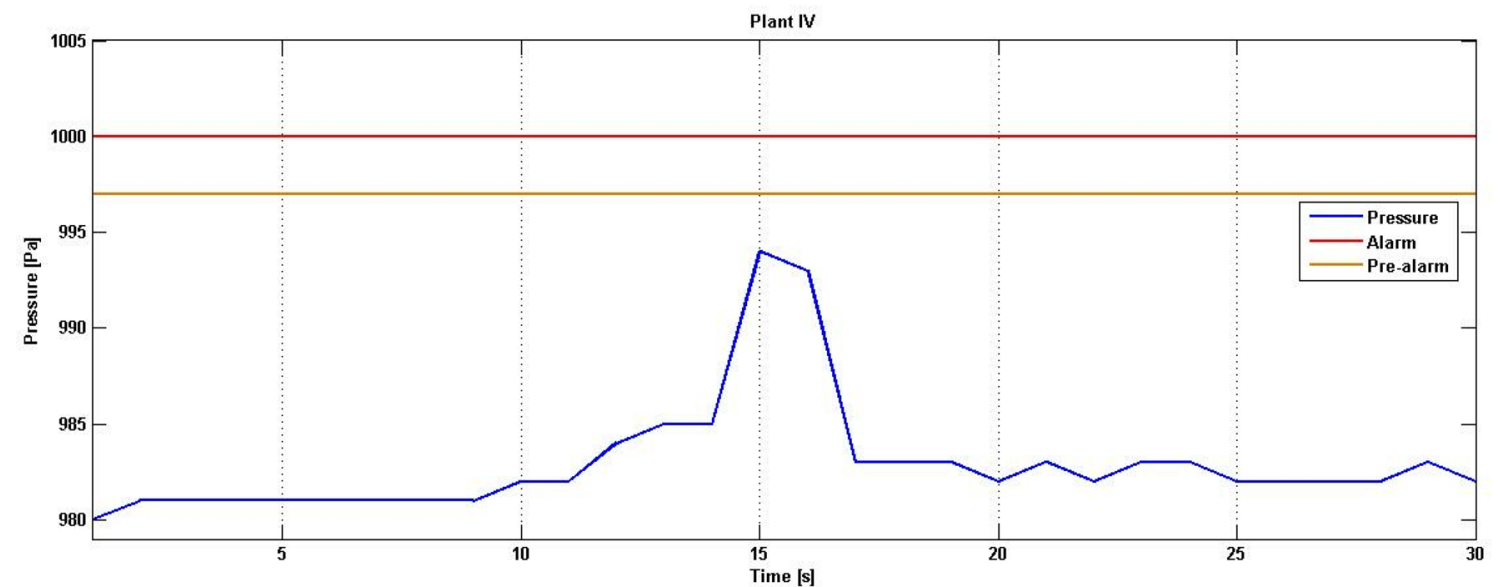

(b)

Fig. 8 Measuring for the selected sensor node (plant 4): a temperature alarm and $\mathbf{b}$ pressure

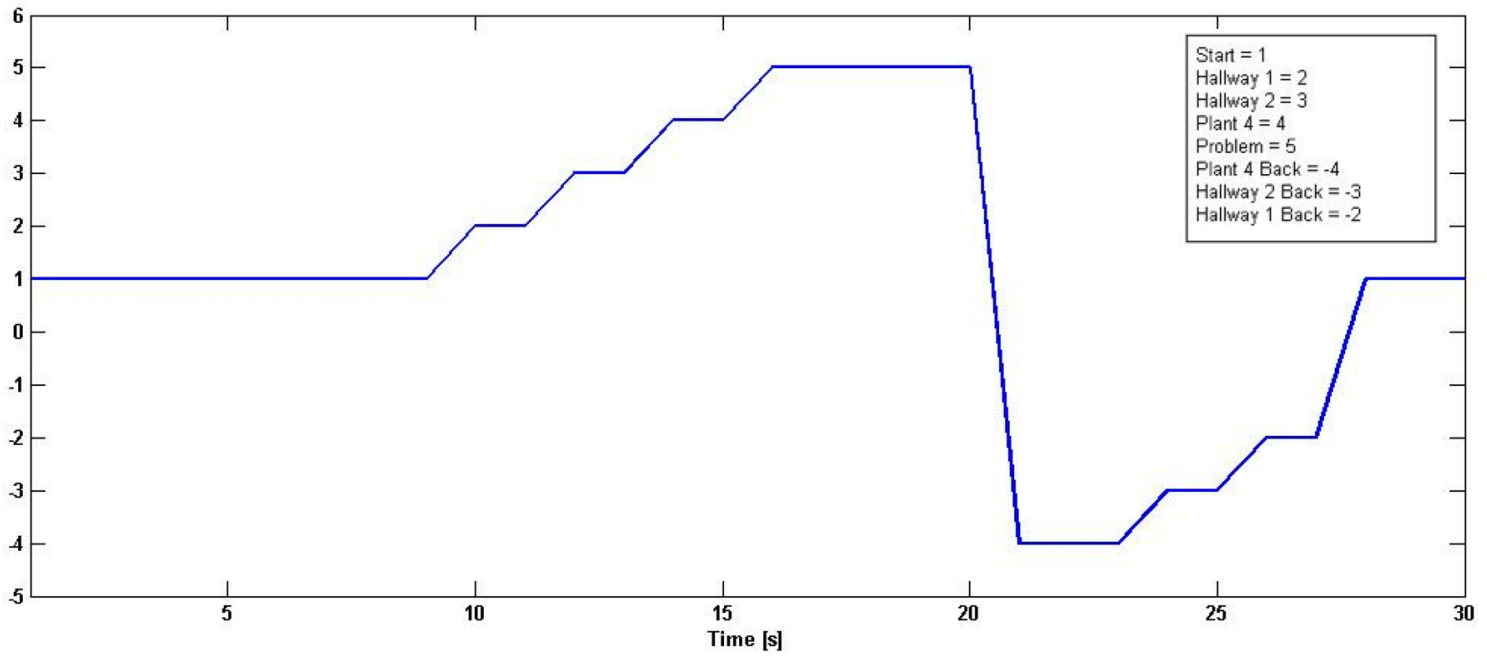

Fig. 9 Inspection mobile robot states in case of alarm detection in plant 4 


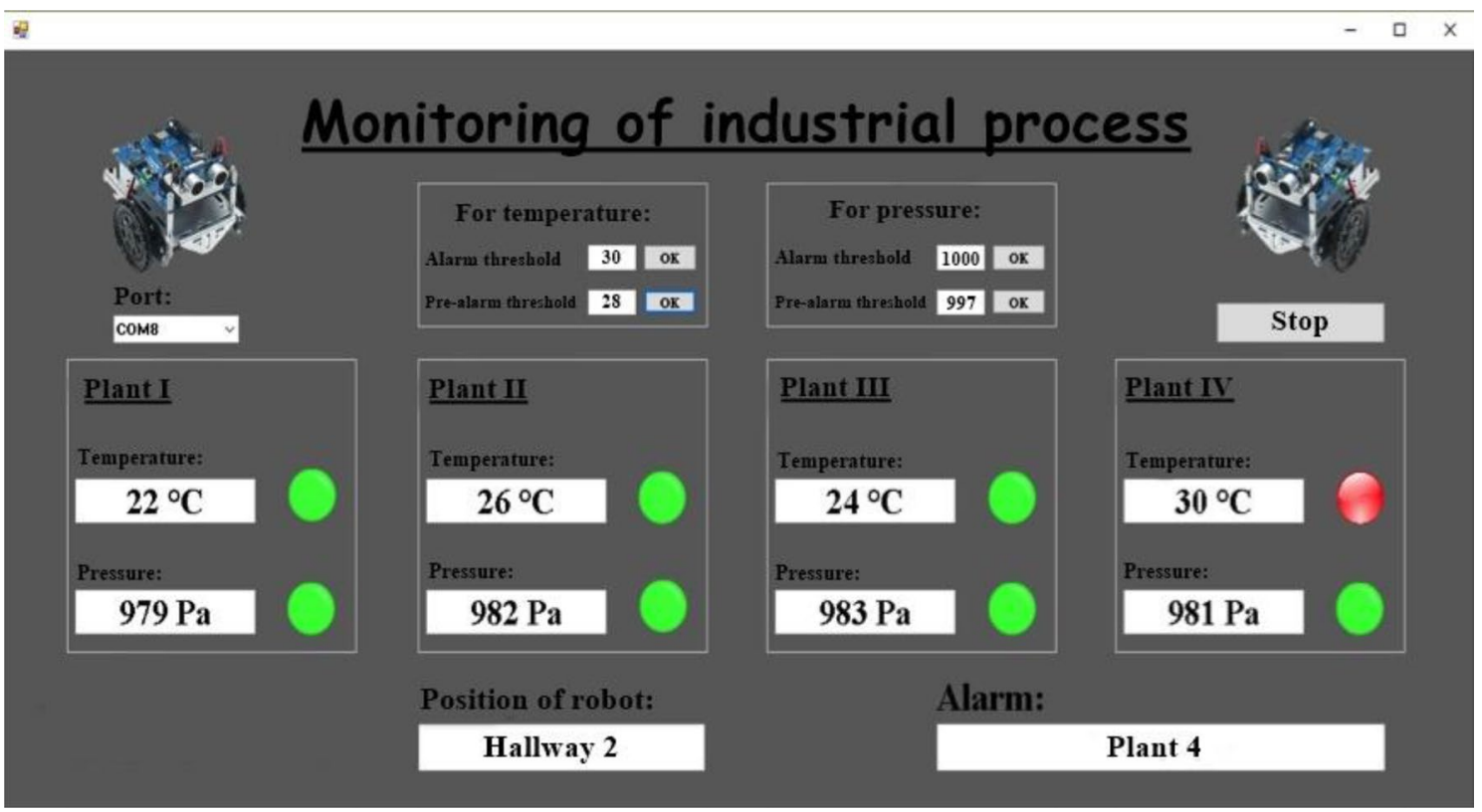

Fig. 10 Monitoring of HWSN based industrial pilot platform

\section{Conclusion}

The industry environmental conditions and equipment monitoring is very important in the smart factories in order to monitor potential threats or predict potential quality issues. The prompt detection and diagnosis of anomalies could be essential to ensure loss prevention and recovery. This research focuses on alarm detection and monitoring of cyber-physical system using the advantages of the hybrid wireless sensor network. We propose the mobile robot navigation technique based on wireless sensor nodes to define the target point for the mobile robot inspection task. The developed system is modular as it can be easily implemented for any type of robotic systems and the working environment in industrial using scalability options.

The realization and the application of the WSN in industrial still need much more experiment and test. Our future work is based on RFID based path planning for collaborative mobile nodes for hybrid WSN consisting of a large number of stationary nodes and a few mobile nodes.

\section{Compliance with ethical standards}

Conflict of interest The authors declare that they have no conflict of interest.

\section{References}

1. Willliam MD (2014) Industry 4.0-smart manufacturing for the future. Germany Trade \& Invest, Berlin

2. Working Group, acatech (2013) Securing the future of German manufacturing industry: recommendations for implementing the strategic initiative INDUSTRIE 4.0. Final report of the Industrie 4.0, 2013

3. Bahrin MAK, Othman MF, Azli NHN, Talib MF (2016) Industry 4.0: a review on industrial automation and robotic. Jurnal Teknologi (Sci Eng) 78(6-13):137-143

4. Michael R, Markus L et al (2015) Industry 4.0: the future of productivity and growth in manufacturing industries. Boston Consulting Group, Boston

5. Burke R, Mussomeli AM, Laaper S, Hartigan M, Sniderman B (2017) The smart factory-responsive, adaptive, connected manufacturing. Deloitte University Press, Westlake

6. Coltin B, Veloso M (2010) Mobile robot task allocation in hybrid wireless sensor networks. In: IEEE/RSJ international conference on intelligent robots and systems (IROS), pp 2932-2937

7. Mikhaylov K, Tervonen J, Heikkilä J, Känsäkoski J (2012) Wireless sensor networks in industrial environment: real-life evaluation results. In: IEEE 2 nd baltic congress on future internet communications, Latvia

8. Jonathan M-G, Trinidad S-R, Juan AGG, Aránzazu D, Fernando G-B, Raúl J (2017) A wireless sensor system for real-time monitoring and fault detection of motor arrays. Sensors 17:469. https ://doi.org/10.3390/s17030469

9. Lu S, Zhang Y, Su Y (2017) Mobile robot for power substation inspection: a survey. IEEE/CAA J Autom Sin. https://doi. org/10.1109/JAS.2017.7510364

10. Li YY, Parker LE (2008) Intruder detection using a wireless sensor network with an intelligent mobile robot response. In: IEEE southeast conference 2008 Huntsville, Alabama

11. Zhang L, Lim C-C, Chen Y (2014) H R Karimi (2014) Tracking mobile robot in indoor wireless sensor networks. Math Probl Eng 837050:8. https://doi.org/10.1155/2014/837050 
12. Cho Y, Youn JH (2006) Wireless sensor-driven intelligent navigation robots for indoor construction site security and safety. In: 23rd international symposium on automation and robotics in construction (ISARC), Tokyo, Japan, October 3-5, 2006, pp 493-499

13. Lambrou TP (2011) Collaborative area monitoring using wireless sensor networks with stationary and mobile nodes, Ph.D. thesis, University of Cyprus

14. Us T, Jensen N, Lind M, Jørgensen SB (2011) Fundamental principles of alarm design. Int J Nucl Safety Simul 2(1):44-51

15. Sun X, Zhuang A, Wang B, Li T, Liu Q (2014) INFLE: an improved neighbor-based fuzzy logic event detecting algorithm for wireless sensor networks. Int J Future Gener Commun Netw 7(3):13-24

16. Amiruddin AAAM, Zabiri H, Taqvi SAA, Tufa LD (2018) Neural network applications in fault diagnosis and detection: an overview of implementations in engineering-related systems. Neural Comput Appl. https://doi.org/10.1007/s00521-018-3911-5

17. Ammar MB, Souissi R (2014) A new approach based on wireless sensor network and fuzzy logic for forest fire detection. Int J Comput Appl (0975 - 8887) 89(2):48-55

18. Qureshi KN, Abdullah AH (2014) Adaptation of wireless sensor network in industries and their architecture, standards and applications. World Appl Sci J 30(10):1218-1223
19. Banjanovic-Mehmedovic L, Husejnovic A, Bosankic I, Kasapovic $S$ (2016) Monitoring of cooperation between autonomous vehicles in roundabout environment. In: 3rd international conference New technologies-2016 development and applications. Bosnia and Herzegovina, pp 377-383

20. Gueaieb W, Miah MS (2008) An intelligent mobile robot navigation technique using RFID technology. IEEE Trans Instrum Meas 57(9):1908-1917

21. Park S, Enriquez G, Hashimoto S (2011) Autonomous mobile robot navigation systems using RFID and their applications. In: Handbook of position location: theory, practice, and advances. Wiley, pp 1023-1054. https://doi.org/10.1002/9781118104750. ch30

22. Wei SS, Ramanathan KC (2012) RFID based intelligent navigation methodology of a nonholonomic indoor autonomous mobile robot. J Appl Sci 12(23):2376-2382

23. Berns K, von Puttkamer E (2009) Autonomous land vehicles, steps toward sevice robots. Vieweg + Teubner, Verlag, Berlin

Publisher's Note Springer Nature remains neutral with regard to jurisdictional claims in published maps and institutional affiliations. 